\title{
Incidence and non-union rate of tibia fractures in adults with Osteogenesis Imperfecta: a retrospective cohort study of an expert clinic of 402 patients with $\mathbf{4 2}$ fractures.
}

Simone Munk ( $\nabla$ simoneambermunk@gmail.com )

Isala Zwolle https://orcid.org/0000-0002-4022-2797

Gerrit Jan Harsevoort

Isala Klinieken: Isala

Koert Gooijer

Isala Klinieken: Isala

Mireille Edens

Isala Klinieken: Isala

Antonius Franken

Isala Klinieken: Isala

Guus Janus

Isala Klinieken: Isala

\section{Research Article}

Keywords: Osteogenesis Imperfecta, tibia fracture, non-union, adult

Posted Date: February 17th, 2022

DOI: https://doi.org/10.21203/rs.3.rs-1329760/v1

License: (c) (i) This work is licensed under a Creative Commons Attribution 4.0 International License.

Read Full License 


\section{Abstract}

\section{Purpose}

Tibia fractures are the most common fractures seen in adults and also lead to the most non-unions. Osteogenesis Imperfecta (OI) is characterized by an increased bone fragility and a higher risk of fractures. No previous studies have been published concerning the incidence of tibia fractures and nonunions in adults with OI. This study aims to give an overview of the incidence of tibia fractures and nonunions in adult Ol patients.

Methods

It is a retrospective, descriptive study where all medical charts of adult patients in the Ol database of our Ol expert clinic were analyzed for tibia fractures between 2008 and 2020. Tibia fracture incidence, nonunion rate, treatment modality and potential risk factors were determined.

\section{Results}

The database consisted of 402 patients of which 34 patients had suffered one or more tibia fractures, resulting in 42 fractures. The incidence of a tibia fracture in Ol adults is 870 per 100.000 person-years. Two out of 42 fractures led to a non-union (5\%). It was not possible to adjust for risk factors or type of treatment.

Conclusion

There is a higher incidence of tibia fractures in Ol patients, but a comparable non-union rate to the general population. With only 2 non-unions it is not possible to draw conclusions on the influence of risk factors or treatment of tibia fractures in OI.

\section{Introduction}

Osteogenesis Imperfecta (OI), also known as brittle bone disease, is a genetic disorder most often caused by alterations in type I collagen that encompasses a heterogenous group of inherited bone dysplasias ${ }^{[1]}$. Among other abnormalities, it impacts the quality of the bone structure, resulting in an increased risk of long bone fractures and skeletal deformation ${ }^{[1]}$. Sillence made a classification based on clinical variety including type I-IV. In 2009 type V was added to the classification. The types have a variety in bone fragility with type II being the worst and lethal prenatally, followed by type III showing skeletal deformity and type $\mathrm{V}$, IV and I, being the least at risk for fractures ${ }^{[2]}$.

Treatment of fractures in $\mathrm{Ol}$ is difficult due to the porous quality of the bone and a wide range in skeletal deformities $^{[1,3]}$. Furthermore, it can lead to complications and failure because of its challenging nature ${ }^{[4]}$. Moreover, a study on femoral fractures in Ol adults concluded that these fractures led to non-union after 
treatment in up to $20 \%$ of the fractures ${ }^{[5]}$, which increases the difficulty of treatment of fractures in $\mathrm{Ol}$ patients.

In the general population, tibia fractures are the most common long bone fractures, which also result in a non-union more often than other long bone fractures. The non-union rates in published studies are varied due to scarce existing literature and different definitions of non-union ${ }^{[6.8]}$. Up until now, as to our knowledge, there has been no research on treatment of tibia fractures and the non-union rate in Ol adults.

Thus, tibia fractures already have a high risk of developing a non-union. With the knowledge of femoral fractures often leading to non-union in Ol adults ${ }^{[5]}$, as a consequence, we expect that tibia fractures in OI will lead to even more non-unions. This descriptive study follows Goudriaan et al. ${ }^{\left[{ }^{[3}\right.}$ in giving an overview on the incidence and non-union at our expert clinic in Zwolle, the Netherlands, but now in tibia fractures in Ol adults. Furthermore, it aims to give an advice on treatment of tibia fractures in Ol.

\section{Method}

\section{Study design and setting}

We performed a retrospective observational cohort study in the Ol expert clinic for adults in Zwolle, the Netherlands. For this study, data were retrieved from January 2008 until February 2020.

\section{Study population}

For this study, the medical data of all OI patients above 18 years were retrieved. This resulted in $402 \mathrm{OI}$ patients in the study period from 2008 to 2020. Inclusion criteria were the diagnosis of Ol and being 18 years or older at the time of tibia fracture (Table 1). Exclusion criteria were the use of corticosteroids, a second genetic syndrome or primary osseous tumor or metastatic disease, since these could influence the risk of fracture and non-union ${ }^{[7]}$. Death before finishing treatment was also an exclusion criterion since union and adequacy of treatment could not be assessed (Table 1).

\section{Data and data collection}

Data collection was aided by using a medical business intelligence tool (CT/cue). The files of all patients were searched with CT/cue by using the following key words (in Dutch): lower leg fracture, tibia fracture, intramedullary pen tibia and intramedullary pen lower leg, plate fixation tibia and plate fixation lower leg.

\section{Data}

As in Goudriaan et al. ${ }^{[5]}$, data selected from the patients files included demographic characteristics and the type of Ol: both genetic and clinical type according to Sillence were included. Furthermore, the type of the fracture according to the AO/OTA Fracture and Dislocation Classification ${ }^{[9]}$, treatment of the fracture (conservative and operative) including outcome (union, non-union) and complications (infection, malposition, failure of osteosynthesis) were recorded. In addition, potential risk factors for non-union 
were identified, including bisphosphonate use, smoking, nutritional deficiency, vitamin D deficiency, mobilization status, metabolic disease and endocrine pathology. Union was defined as the presence of bridging callus in at least three of four cortices, evaluated on two transverse levels on radiographic imaging. Non-union was defined as non-radiographic changes to union or the absence of bridging callus of two or more cortices, evaluated on radiographs in two transverse levels, for at least 6 months after treatment ${ }^{[5,10]}$.

In the case of missing data, patients were asked for permission to retrieve the missing documentation from other hospitals. Information was only obtained when the patients permission for requesting data for research was documented.

Table 1

In- and exclusion criteria

\begin{tabular}{|ll|}
\hline Inclusion & Exclusion \\
\hline Tibia fracture & No tibia fracture \\
\hline $\begin{array}{l}\text { Age } \geq 18 \text { years } \\
\text { Osteogenesis }\end{array}$ & Age $<18$ years \\
\hline & Use of corticosteroids \\
& $\begin{array}{l}\text { Death before full healing of fracture } \\
\text { disease }\end{array}$ \\
\hline
\end{tabular}

\section{Statistical methods}

This study contains a relatively small number of subjects with too little data to analyze statistically: therefore, a descriptive study was performed. Descriptive statistics were used to analyze the results using SPSS. Categorical variables were expressed as percentage and metric variables as mean and standard deviation.

The incidence rate was calculated over all 402 patients and the 12 years of inclusion $(2008-2020)$. The incidence was calculated on the amount of fractures, not individuals.

\section{Ethics}

The Medical Ethics Committee of the Isala Hospital, Zwolle, The Netherlands, approved the study protocol and provided a non-WMO (Medical Research Involving Human Subjects Act) waiver (METC no. 200638). All patients had provided written consent for reading their medical records, for obtaining additional records from other institutions and use of this information for research.

\section{Results}


Of the 402 patients with OI in the database during the stated study period, 132 patients were selected with potential tibia fracture(s) using CT/cue. The remaining 270 patients were assumed not to have had a tibia fracture since none of the search terms were found in their files.

The dossiers of these 132 patients were reviewed in full by one researcher (SAM). 98 patients were excluded (Fig. 1) and 34 patients met the criteria of this study and were included. The demographics of the participants are shown in Table 2.

Six of the participants had more than one tibia fracture over time, which were included separately, resulting in a total of 42 fractures. Of these fractures, 2 resulted in non-union after treatment.

Table 2

Demographic characteristics of all subjects with one or more tibia fractures

\begin{tabular}{|ll|}
\hline & $\begin{array}{l}\text { All subjects } \\
\mathrm{N}=\mathbf{3 4}\end{array}$ \\
\hline Age (years) & $41[32 ; 61]$ \\
\hline Gender (male, \%) & $17(50)$ \\
\hline Smoking $(\mathrm{n}, \%)$ & $10(29)$ \\
\hline OI type $(\mathrm{n}, \%)$ & \\
\hline I & $22(65)$ \\
\hline III & $6(17)$ \\
\hline IV & $5(15)$ \\
\hline V & $1(3)$ \\
\hline Data are presented as: median [quartile 1; quartile 3] or number of patients (percentage) \\
\hline
\end{tabular}

\section{Incidence of tibia fracture and non-union}

There were 42 fractures in the cohort of 402 Ol patients recorded from 2008 to 2020 . This results in an incidence of tibia fractures of $870 / 100.000$ annually. Two out of 42 fractures (5\%) developed into a nonunion. The incidence for non-union is $38 / 100.000$ annually.

\section{Influence of Ol type on healing rate}

Twenty-six (62\%) tibia fractures were seen in Sillence type I patients and respectively 7 (17\%) and 8 (19\%) in type III and IV OI patients, and $1(2 \%)$ in type V. Of the 42 analyzed fractures, 2 resulted in a nonunion (Table 3). One of these fractures was a proximal fracture in an OI type I patient, the other nonunion pertained a distal fracture in OI type III.

\section{Influence of treatment on healing rate}


For OI type I, only 1 out of 26 fractures (4\%) resulted in a non-union. This proximal fracture was initially treated with a plate fixation. The other non-union was a distal tibia fracture in a type III OI patient, being 1 out of 7 fractures (14\%). As treatment, a rushpen was removed and a titanium elastic nail (TEN) was implanted. With no evidence of healing, an additional cast immobilization was initiated with no result of bone healing. Since the patient is wheelchair bound and had little pain, eventually the pseudarthrosis was accepted and the patient uses a brace for support (Fig. 2).

Looking from another perspective, 1 out of 5 (20\%) plate fixations for proximal tibia fractures led to a non-union. As for distal intramedullary nailing, 1 out of $3(33 \%)$ led to a non-union. Conservative treatment alone (18 fractures) never led to a non-union, independent of location of the fracture.

Table 3

Characteristics of patients with non-unions

\begin{tabular}{|llllll|}
\hline & Gender & Age at fracture & Type Ol & Type fracture & Primary treatment \\
\hline 1 & $\mathrm{M}$ & 19 & $\mathrm{I}$ & Proximal tibia & Plate fixation \\
\hline 2 & $\mathrm{M}$ & 22 & $\mathrm{III}$ & Distal tibia & Intramedullary fixation \\
\hline$M=$ male & & & & \\
\hline
\end{tabular}

\section{Discussion}

In our study, the incidence of tibia fractures is $870 / 100.000$ annually in Ol adults where we included a total number of 42 tibia fractures in 402 patients (10\%), with 2 non-unions (5\%). To our knowledge, this is the first study that describes the incidence and number of non-unions in tibia fractures in adults with Ol. We also aimed to draw conclusions as to whether the treatment or type of OI has any impact on the risk of non-union, but with only two non-unions with different treatments, this was not feasible.

Tibia fractures are the most common fractures in the general population. There is a recent study that estimates the incidence of tibia fractures to be between 6-101/100,000 per year in the general population [11]. The incidence of tibia fractures in this Ol population is $870 / 100.000$ per year, thus higher than the general population. However, both Hemmann et al[11] and the present study most likely give an underestimation of reality. In Hemmann they only include in-hospital patients, and in this study not all fractures of the OI population we studied might be known in our patient files: if treated elsewhere and not communicated with the expert clinic, the fracture would not have been traceable for this study. In addition the used exclusion criteria and the use of specific search terms in the selection of participants could also have led to an underestimation of incidence.

Tibia fractures are also seen with the most non-unions, even in the general population, ranging from $1 \%$ up to $23 \%[7,8,12,13]$. Our findings of $5 \%$ show a comparable non-union rate. The outcome differs from our expectation where we assumed the non-union rate would be higher in OI. Important to note is that a tibia fracture is often the result of a high energy trauma $[7,8,13]$. One study specifically on tibia shaft fractures 
showed the mechanism of injury and amount of soft tissue damage indeed heightened the risk on nonunion ${ }^{[14]}$. In our study, the fractures were all the result of low energy trauma. Therefore, the results are not directly comparable: it might be the case that if corrected for intensity of trauma, the non-union rate would be lower than in the known literature on the general population.

\section{Strengths and Limitations}

This is a retrospective study where patient data was searched by using specific terms. Therefore, we cannot exclude the possibility of having missed fractures in the database. In addition, not all tibia fractures might be documented in our database, especially the ones that were treated successfully elsewhere. However, since Zwolle is the expert clinic on Ol, it is to be expected that non-unions would have been referred to or at least discussed with our clinic and therefore would most likely be in our database.

By stating a clear definition of non-union that can objectively be measured on $\mathrm{x}$-ray, there is no question on whether or not the fracture healed. With consent of all patients, it was possible to retrieve almost all missing data required to determine union.

\section{Patient-dependent factors}

With the retrospective nature of this study, we were able to include risk factors: bisphosphonate use, smoking, nutritional and vitamin D deficiency, mobilization status and other metabolic diseases. Unfortunately, data were incomplete and despite efforts made, not all data could be retrieved since often it was not documented. In addition, there were only two non-unions in the current study, making it impossible to adjust for these risk factors, even if the data would have been complete.

\section{Conclusion}

This study set out the incidence and non-union rate of tibia fractures in the adult OI population known at our expert clinic. Whereas the incidence of tibia fractures is higher in the OI population, the non-union rate is comparable to a general population, although there might be a difference if type of injury and soft tissue damage would be taken into account.

With only two non-unions, we could not draw any hard conclusions as to whether type of OI, type of treatment or risk factors influence the risk of non-union in tibia fractures.

Further research must be done to be able to make an adequate statement, preferably multicenter. With more data, it could be possible to give a useful advice on how to treat a non-union, leading to better care for the OI population.

\section{Declarations}

Funding Not applicable. 
Conflict of interest SAM, GJH, KG, MAE, AAF and GJMJ declare that they have no conflict of interest.

Availability of data and material The data used and analyzed for the current study are available from the corresponding author on reasonable request.

Code availability Not applicable.

Authors contributions SAM, GJH and GJMJ significantly contributed to the literature review, the writing of the manuscript, and the creation of diagrams. KG, MAE and AAF participated in the conceptual development of the manuscript and revised the article for intellectual content. All authors read and approved the final manuscript.

Acknowledgements Not applicable.

Approval by the local ethics committee METC Isala, Zwolle, The Netherlands. Registration number 200638. Date of issue: august 31, 2020.

Consent to participate All participants approved to use medical files and if needed additional information from other hospitals.

Consent for publication All data and images used were with consent of the participants.

\section{References}

1. Marini JC, Forlino A, Bachinger HP, et al. Osteogenesis imperfecta. Nat Reviews Disease Primers. 2017;3:1-19. doi:10.1038/nrdp.2017.52.

2. Van Dijk FS, Sillence DO. Osteogenesis imperfecta: Clinical diagnosis, nomenclature and severity assessment. Am J Med Genet A. 2014;164A(6):1470-81. doi:10.1002/ajmg.a.36545.

3. Ralston SH, Gaston MS. Management of osteogenesis imperfecta. Front Endocrinol. 2020;11. doi:https://doi.org/10.3389/fendo.2019.00924.

4. Chiarello E, Donati $D$, Tedesco G, et al. Conservative versus surgical treatment of osteogenesis imperfecta: A retrospective analysis of 29 patients. Clin Cases Mineral Bone Metabolism. 2012;9(3):191-4.

5. Goudriaan WA, Harsevoort GJ, van Leeuwen M, Franken AA, Janus G. Incidence and treatment of femur fractures in adults with osteogenesis imperfecta: An analysis of an expert clinic of 216 patients. Eur J Trauma Emerg Surg. 2020;46(1):165-71. doi:10.1007/s00068-018-1005-9 [doi].

6. Ekegren CL, Edwards ER, de Steiger R, Gabbe BJ. Incidence, costs and predictors of non-union, delayed union and mal-union following long bone fracture. Int J Environ Res Public Health. 2018;15. doi:10.3390/ijerph15122845.

7. Stewart SK. Fracture non-union: A review of clinical challenges and future research needs. malaysian Orthop J. 2019;13(2):1-10. doi:http://doi.org/10.5704/MOJ.1907.001. 
8. Mills LA, Aitken SA, Simpson A. The risk of non-union per fracture: Current myths and revised figures form a population of over 4 million adults. Acta Orthop. 2017;88(4):434-9. doi:10.1080/17453674.2017.1321351.

9. Meinber EG, Agel J, Roberts CS, Daram MD, Kellam JF. Fracture and dislocation classification compendium - 2018. J Orthop Trauma. 2018;32:1. doi:10.1097/BOT.0000000000001063.

10. Frolke J, Patka P. Definition and classification of fracture non-unions. Injury. 2007;38:19. doi:https://doi.org/10.1016/S0020-1383(07)80005-2.

11. Hemmann P, Friederich $M$, Korner D, Klopfer T, Bahrs C. Changing epidemiology of lower extremity fractures in adults over a 15-year period - a national hospital discharge registry study. BMC Musculoskelet Disord. 2021;22. doi:https://doi.org/10.1186/s12891-021-04291-9.

12. Tzioupis C, Giannoudis PV. Prevalence of long-bone non-unions. Injury. 2007;38(10):3. doi:10.1016/s0020-1383(07)80003-9.

13. Nandra R, Grover L, Porter K. Fracture non-union epidemiology and treatment. Trauma. 2015;0(0):19. sagepub.co.uk/journalsPermission.nav. doi:10.1177/1460408615591625.

14. Karladani H, Granhed H, Karrholm J, Styf J. The influence of fracture etiology and type on fracture healing: A review of 104 consecutive tibial shaft fractures. Arch Orthop Trauma Surg. 2000;121(6):325-8. doi:10.1007/s004020000252.

\section{Figures}

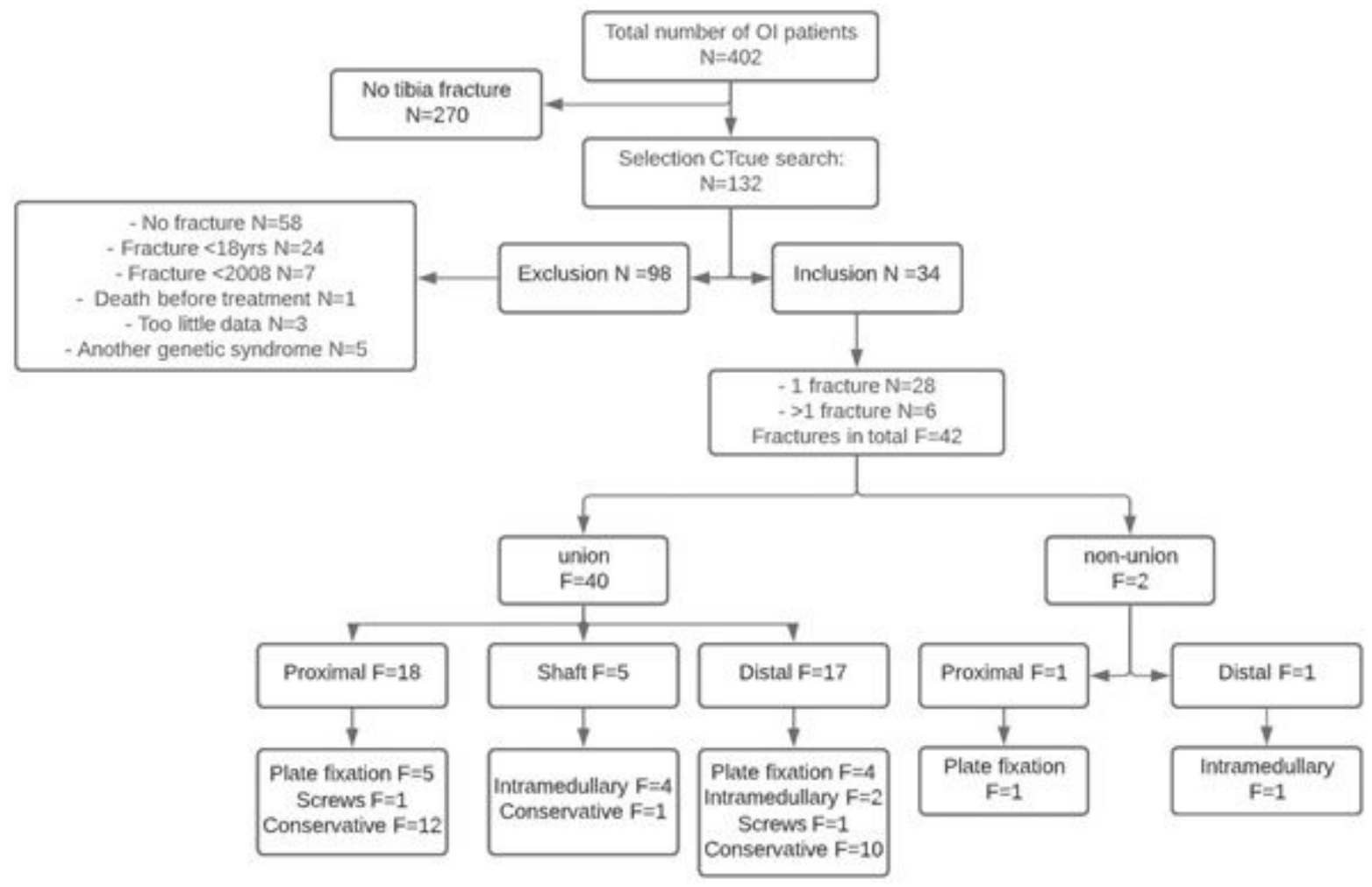

Figure 1 
flowchart of participant and fracture selection, union classification and treatment of fractures

Legend: $\mathrm{N}=$ number of participants. $\mathrm{F}=$ number of fractures

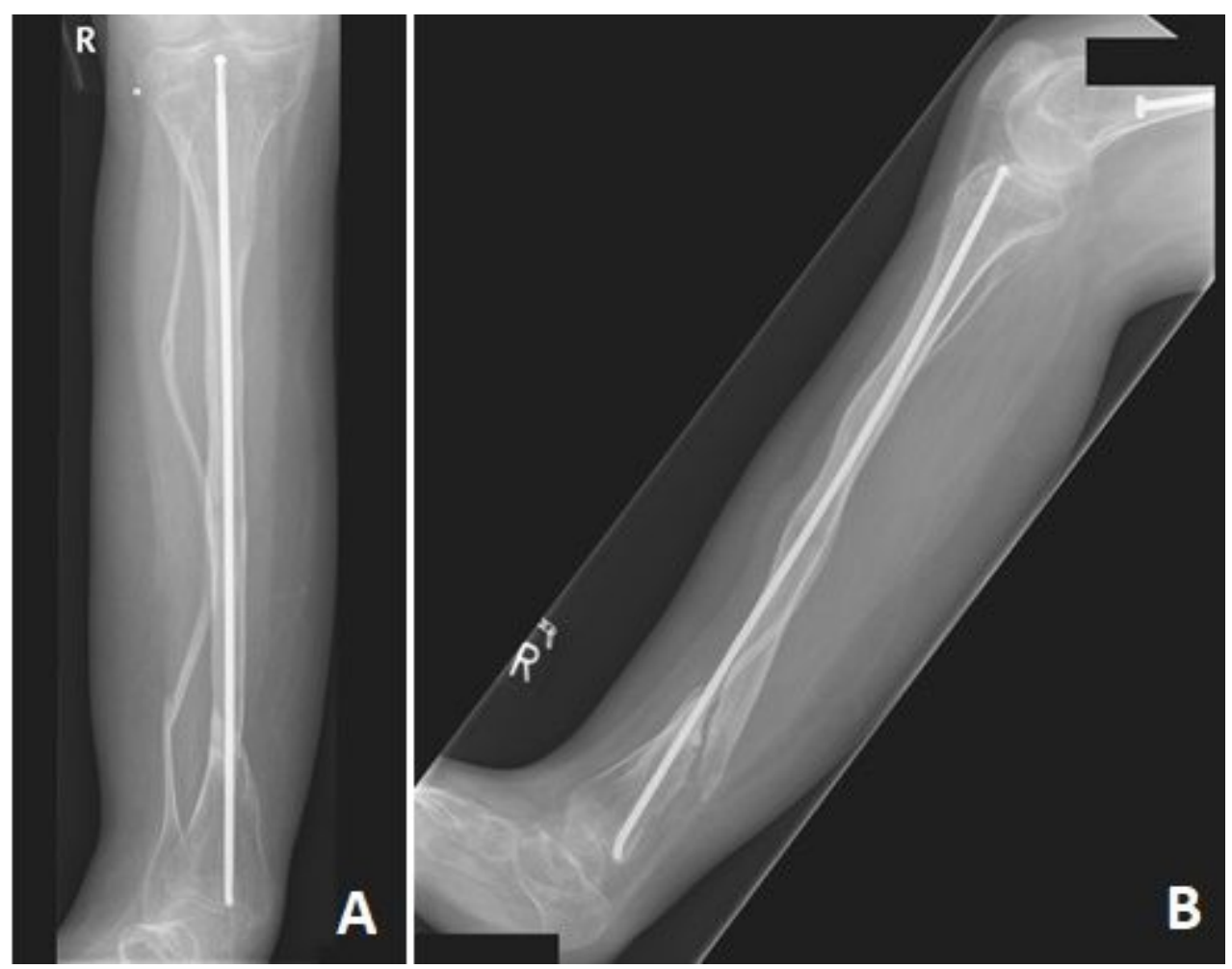

Figure 2

non-union distal tibia fracture, 2 years after initial treatment

a. AP en b. lateral view 